\title{
Modificaciones térmicas en Lima: Análisis de la isla de calor urbana
}

\author{
Thermal modifications in lima: analysis of urban heat island
}

\author{
Patricia M. Barros P. ${ }^{1}$; Ever Menacho C. ${ }^{2}$
}

\begin{abstract}
Resumen
La expansión urbana de la ciudad de Lima y su crecimiento sustituyen el suelo natural por materiales de construcción originando la alteración del ambiente circundante, afectando principalmente al balance de energía, lo cual ocasiona el incremento de la temperatura del aire. La isla de calor urbana corresponde al aumento de la temperatura del aire en la ciudad, por origen antrópico, en comparación con su entorno inmediato de carácter natural y rural. Se resalta que el presente estudio es un caso puntual en el tiempo, teniendo como principal objetivo determinar la existencia de la isla de calor urbana en 27 distritos de Lima y establecer su relación con variables condicionantes que intervienen en su forma e intensidad. A partir del uso de data proporcionada por estaciones meteorológicas fijas y por la metodología de los transectos, se creó una base de datos, cuya integración en un Sistema de Información Geográfica permitió elaborar la distribución de la temperatura del aire a las 16:00 h (hora local) y a las 19:00 h (hora local), determinándose la presencia de dos núcleos cálidos concéntricos, que bordean $\operatorname{los} 27,2{ }^{\circ} \mathrm{C}$ a las $16: 00$ horas y de un núcleo cálido concéntrico, que bordea los $26,3{ }^{\circ} \mathrm{C}$ a las 19:00 horas. Finalmente, con la aplicación del análisis de regresión múltiple por pasos, se determinó la influencia de las variables de carácter geográfico y urbano del área de estudio en las dos horas de análisis, evidenciándose que a las 16:00 h la isla de calor se encontró condicionada por la altitud, la densidad poblacional, la densidad urbana media, la densidad urbana baja y el uso residencial del suelo, mientras que a las 19:00 $\mathrm{h}$ fue condicionada por la densidad urbana media, la altitud, el uso residencial del suelo, la densidad urbana baja y la distancia a fuentes de agua.
\end{abstract}

Palabras clave: análisis de regresión múltiple por pasos; isla de calor urbana; temperatura del aire; transectos; urbanización.

\begin{abstract}
Urban expansion of Lima city and its growth replaced natural soil with construction materials, causing the alteration of the surrounding environment, which affects the energy balance, producing the increase of air temperature. The urban heat island corresponds to the increase of air temperature in the city, by anthropic origin, in comparison to its immediate surroundings of nature and rural environment. It is highlighted that the present study is a punctual case in time, and its main objective was to determinate the urban heat island in 27 districts of Lima and to establish its relationship with conditioning variables involved in its form and intensity. Using the information provided by fixed weather stations and transects methodology a database was created, whose integration to a geographic information system allowed the elaboration of the air temperature distribution at 16:00 h (local hour) and 19:00 $\mathrm{h}$ (local hour), determining the presence of two concentric warm nuclei, bordering the $27,2{ }^{\circ} \mathrm{C}$ at $16: 00$ and a concentric warm core, which borders the $26,3{ }^{\circ} \mathrm{C}$ at 19:00. Finally, with the implementation of the analysis of multiple regression by steps, it was possible to determine the influence of the geographical and urban variables of the area of study within the two hours of analysis, showing that at 16:00 $\mathrm{h}$. the heat island was conditioned by altitude, population density, low urban density, average urban density, and the residential land use, while at 19:00 h. was conditioned by average urban density, residential land use, low urban density and distance to water sources.
\end{abstract}

Keywords: multiple regression analysis by steps; urban heat island; air temperature; transects; urbanization.

\section{Introducción}

La urbanización se ha incrementado de una manera considerable durante estos últimos años. En el Perú, de acuerdo con el Instituto Nacional de Estadística e Informática - INEI (2007), la población urbana representó un 75,9\% de la población nacional, 5,8\% más que la que representó en el 2003 y específicamente en Lima y Callao se concentró aproximadamente el 40,7 $\%$ de la población urbana en un área de $2.817 \mathrm{~km}^{2}$. El proceso de urbanización tiene un gran impacto en las 
propiedades de la superficie del suelo y en la atmósfera circundante. El cambio de la composición de la superficie natural del suelo por actividades antropogénicas afecta principalmente al balance de energía por la predominancia de materiales de alta capacidad calorífica como lo son el hormigón, el asfalto y el cemento, entre otros. La alteración del balance de energía en una ciudad trae como consecuencia inmediata el incremento de la temperatura, la cual se ve además intensificada por la emisión de contaminantes hacia la atmósfera, provenientes de las actividades humanas. El incremento de temperatura producido en la ciudad, debido a la expansión urbana, en relación con el espacio rural circundante da lugar a un fenómeno conocido como "isla de calor urbana" (ICU). Los primeros estudios teóricos referentes a la isla de calor datan de los años 1818 y 1868 , que corresponden respectivamente a los trabajos del británico Luke Howard y del francés Emilien Renou, donde plantean la diferencia de la temperatura del aire entre la ciudad y los campos circundantes.

Debido al acelerado proceso de urbanización en Lima, el presente trabajo de investigación pretendió ampliar el conocimiento de este fenómeno ambiental en Lima metropolitana ya que un incremento de la temperatura, debido al efecto de la isla de calor, influye en el consumo de energía, en la contaminación atmosférica y en el confort climático. Asimismo, un estudio sobre la isla de calor urbana proporciona el conocimiento de las interacciones entre el clima y la ciudad de Lima, y a pesar de que esta ciudad es la más grande e importante del país, los estudios relacionados con el clima urbano son casi inexistentes, por lo que el estudio de la ICU en Lima Metropolitana puede guiar las actividades relacionadas con la gestión ambiental, los compromisos y estrategias de la planificación y ordenamiento territorial de la ciudad, debido a su gran utilidad para mejorar el confort climático, la calidad del aire y consecuentemente la calidad de vida de los ciudadanos.

El área de estudio solo consideró 27 distritos de Lima Metropolitana, exceptuando los sectores norte y sur, y tuvo como objetivo principal determinar la presencia de la isla de calor urbana a distintas horas y establecer su relación con las variables que condicionan su forma e intensidad, considerándose los siguientes objetivos específicos: 1) Identificar la presencia de la isla de calor; 2) Determinar la intensidad de la isla térmica y sus variaciones a diferentes horas; 3) Precisar la distribución espacial de las variables condicionantes (densidad poblacional, uso de suelo, densidad urbana, distancia a fuentes de agua y altitud) en los distritos analizados y 5) Analizar la influencia de las variables condicionantes en el desarrollo de la isla de calor del área de estudio.

\section{Materiales y métodos}

Para detectar y caracterizar el fenómeno de la isla de calor en una ciudad, se pueden utilizar distintos métodos, entre los cuales destacan: (a) la comparación de registros de temperatura en estaciones urbanas y rurales, (b) el análisis de series históricas de temperatura, (c) el método de los transectos, (d) las observaciones aéreas con imágenes satelitales y (e) modelos climáticos meteorológicos.

El presente estudio ha empleado la metodología de los transectos para la detección de la presencia de la ICU en el área de estudio, debido a que proporciona una gran cantidad de datos meteorológicos que resuelve el problema de insuficiencia de datos en la ciudad.

Equipos y datos.

Se utilizaron los datos meteorológicos de: (a) la temperatura del aire, (b) la humedad relativa, (c) la velocidad del viento y (d) la dirección del viento, proporcionados por las diferentes instituciones que tienen a cargo las estaciones meteorológicas fijas en la ciudad de Lima (Tabla 1) para poder conocer el comportamiento de estas variables meteorológicas para los días 12 y 19 de marzo del 2009.

Los días de estudio se caracterizaron por tener baja cobertura de nubosidad y presencia de sol. Asimismo, los datos de las estaciones meteorológicas fijas evidenciaron que los valores de temperatura y humedad relativa durante las primeras horas del día disminuyeron hasta un mínimo, que se registró entre las 5:00 y 7:00 horas de la mañana, para luego incrementarse hasta un valor máximo que ocurrió entre las 12:00 y 15:00 horas. En las siguientes horas, la temperatura comenzó su descenso y, posteriormente, se observó una ligera elevación de esta variable durante las 16:00 y 17:00 horas, para el 12 de marzo, y las 18:00 y 20:00 horas para el 19 de marzo. Se resalta que este comportamiento no se observó en la estación meteorológica del hospital central FAP que presentó la temperatura máxima en horas nocturnas, alrededor de las 21:00 horas. Con respecto a la velocidad y dirección del viento, se observó que en la estación Campo de Marte se presentaron los vientos más débiles

Tabla 1. Estaciones meteorológicas fijas utilizadas para la investigación

\begin{tabular}{|l|l|l|l|l|}
\hline \multicolumn{1}{|c|}{ Estación meteorológica } & Institución & \multicolumn{2}{c|}{ Ubicación } & \multicolumn{1}{c|}{ Distrito } \\
\hline Aeropuerto Internacional Jorge Chávez & SENAMHI & $12^{\circ} 01^{\prime} \mathrm{S}$ & $77^{\circ} 06^{\prime} \mathrm{O}$ & Callao \\
Alexander Von Humboldt & UNALM & $12^{\circ} 05^{\prime} \mathrm{S}$ & $76^{\circ} 56^{\prime} \mathrm{O}$ & La Molina \\
Campo de Marte & SENAMHI & $12^{\circ} 04^{\prime} \mathrm{S}$ & $77^{\circ} 02^{\prime} \mathrm{O}$ & Jesús María \\
Hospital Central FAP & SENAMHI & $12^{\circ} 06^{\prime} \mathrm{S}$ & $77^{\circ} 01^{\prime} \mathrm{O}$ & Miraflores \\
Las Palmas & SENAMHI & $12^{\circ} 09^{\prime} \mathrm{S}$ & $77^{\circ} 01^{\prime} \mathrm{O}$ & Santiago de Surco \\
Lima Este & SENAMHI & $12^{\circ} 03^{\prime} \mathrm{S}$ & $76^{\circ} 55^{\prime} \mathrm{O}$ & La Molina \\
\hline
\end{tabular}


$(<2.1 \mathrm{~m} / \mathrm{s})$, predominantemente en dirección $\mathrm{SO}$, y la mayor presencia de calmas (aproximadamente el 80,0 $\%$ ). En la estación hospital central FAP predominaron los vientos $\mathrm{SE}$ con velocidades menores a $3,6 \mathrm{~m} / \mathrm{s}$ y con calmas de 4,2\%. La estación que no presentó periodos de calma y tuvo vientos de intensidad moderada fue la del aeropuerto internacional Jorge Chávez, con una dirección predominante sur. La estación meteorológica Las Palmas presentó vientos con dirección predominantemente sur este alcanzando velocidades de hasta $5,7 \mathrm{~m} / \mathrm{s}$. La estación Lima Este fue la que presentó mayor variabilidad en la distribución del viento, presentando vientos predominantemente de intensidad fuerte $(<11.1$ $\mathrm{m} / \mathrm{s}$ ) y aproximadamente $13,0 \%$ de periodos de calma. Finalmente, la estación Alexander Von Humboldt tuvo vientos con velocidades menores a $3,6 \mathrm{~m} / \mathrm{s}$ (intensidad media débil), con una dirección predominantemente suroeste, y presencia significativa de calmas (alrededor del $40,0 \%)$

Además, durante los días 12 y 19 de marzo del 2009, se realizó el monitoreo de la temperatura del aire y la humedad relativa, a través de la metodología de los transectos. Para ello, se empleó dos estaciones

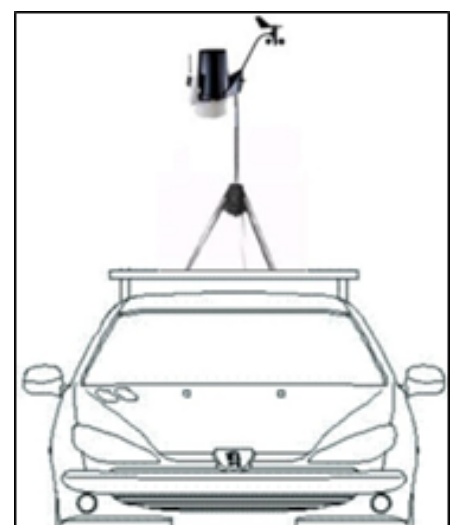

Figura 1. Modelo de la instalación del equipo de monitoreo meteorológicas automáticas móviles, previamente calibradas usando un termómetro de ambiente y datos de humedad relativa del observatorio meteorológico Von Humboldt. Cada estación fue colocada en un soporte y sujetada sobre el techo de un vehículo station vagon de color blanco, quedando a una altura aproximada de $3.00 \mathrm{~m}$ con respecto al suelo, de tal manera que la toma de datos no fuese influenciada por el calor liberado del motor y del tubo de escape. El recorrido de cada transecto se realizó a una velocidad media de $40 \mathrm{~km} / \mathrm{h}$ y se empleó una estación meteorológica automática portátil, previamente montada en el techo de un automóvil station vagon de color blanco a una altura aproximada de $3.00 \mathrm{~m}$ con respecto al suelo, de tal manera que la toma de datos no fuese influenciada por el calor liberado por el motor y el tubo de escape. Finalmente, para el procesamiento de los datos obtenidos se utilizó el Sistema de Información Geográfica ArcGis 9.2.

\section{Metodología \\ Identificación de la presencia de la isla de calor}

Se utilizó el método de los transectos para tomar los datos de temperatura en el área de estudio, debido a que este método es considerado como uno de los más adecuados para conocer la configuración espacial de la temperatura en la ciudad (Cuadrat et al., 1993). Este procedimiento consistió en tomar medidas de temperatura cada 400 metros en dos rutas pre-establecidas de $36 \mathrm{~km}$ aproximadamente a través del uso de automóviles (Fig. 2).

Para la disponibilidad de la temperatura del aire, se realizaron cuatro mediciones durante los días 12 y 19 de marzo. Para cada día de estudio, se consideró dos momentos de análisis: (a) las 16:00 horas, momento cercano a la máxima temperatura y (b) las 19:00 horas, momento cercano a la puesta del sol. Para ello, las mediciones en los dos transectos se iniciaron simultáneamente, a las 15:30

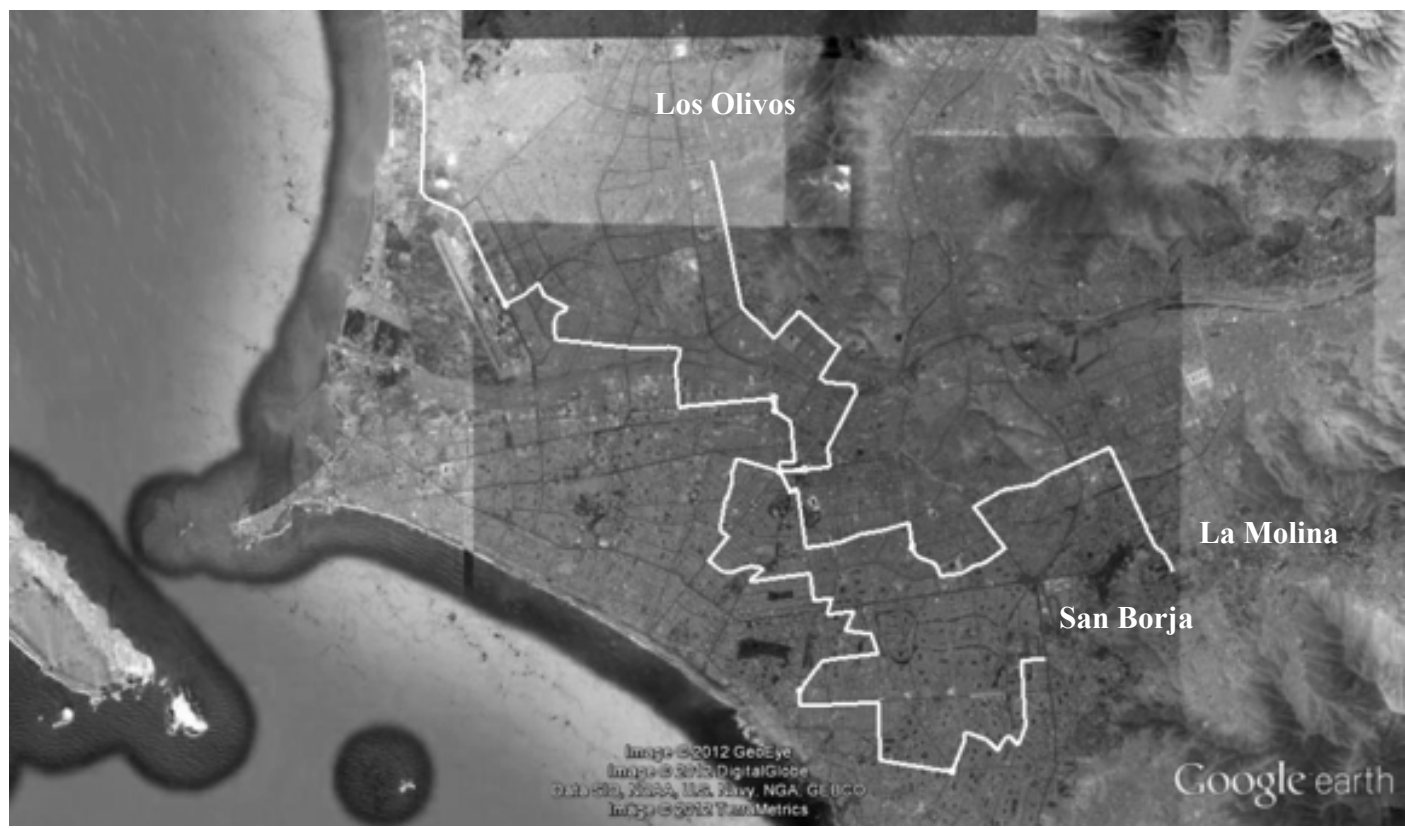

Figura 2. Ruta establecida para los dos transectos realizados 
horas y a las 18:30 horas, con una duración aproximada de una hora y treinta minutos. Los datos generados, en cada hora de análisis, se consideraron como cuasisimultáneos, ya que en las ciudades costeras la influencia marina, por su efecto termorregulador, determina que las amplitudes diarias de temperatura sean reducidas y que las mediciones efectuadas durante un cierto intervalo de tiempo puedan ser consideradas como cuasi-simultáneas (Moreno, 1999).

Posteriormente, se utilizaron los datos de temperatura del aire para las 16:00 horas y las 19:00 horas, de los días 12 y 19 de marzo, de las estaciones meteorológicas fijas próximas a la ruta de los transectos: (a) el aeropuerto internacional Jorge Chávez, (b) Campo de Marte y (c) Alexander Von Humboldt, como valores referenciales para la corrección del desfase temporal (diferencia horaria) de las datos obtenidos durante el recorrido de los dos transectos con respecto a los datos generados por dichas estaciones. Para ello, fue necesario calcular índices de simultaneidad, los cuales permitieron que los puntos monitoreados en un periodo de tiempo se asemejen a los datos obtenidos a las 16:00 h y 19:00 h por las estaciones meteorológicas fijas, anteriormente mencionadas.

Finalmente, se estudió la continuidad o dependencia espacial de la temperatura a través del cálculo del semivariograma omnidireccional. Según Gallardo (2006), el semivariograma representa la semivarianza en función de la distancia, lo que permite describir la variación espacial de una variable en una determinada zona, específicamente en este caso, conocer el comportamiento espacial de la temperatura en el área de estudio. Conociendo el comportamiento espacial de la temperatura para las 16:00 h y las 19:00 h, se aplicó la técnica geoestadística kriging ordinario, mediante el uso del software ArcGis, para interpolar datos en el área de estudio y ser representados cartográficamente.

\section{Determinación de la intensidad de la isla de calor urbana}

La intensidad de la isla de calor urbana en el área de estudio se determinó para cada hora analizada, 16:00 y 19:00 h, mediante la diferencia de la temperatura urbana y la temperatura periurbana del área de estudio $\left(\mathrm{T}_{\text {urbana }}\right.$ - $\mathrm{T}_{\text {periurbana }}$ ), considerándose como áreas periurbanas a algunas zonas pertenecientes a los distritos de Callao, Barranco, Surco y La Molina. La intensidad de la isla de calor en una ciudad puede tener distintos niveles: (a) intensidad débil, hasta $2{ }^{\circ} \mathrm{C}$; (b) intensidad moderada, 2 a $4{ }^{\circ} \mathrm{C}$; (c) intensidad fuerte, 4 a $6{ }^{\circ} \mathrm{C}$ y (d) intensidad muy fuerte, mayores a $6{ }^{\circ} \mathrm{C}$, mencionado por Sarricolea, Aliste, Castro y Escobedo (2008).

\section{Determinación de la distribución espacial de las variables condicionantes de la isla de calor urbana Distribución espacial de las variables geográficas}

La altitud y la distancia a fuentes de agua, como ríos y mar, del área en estudio se obtuvieron a partir de la información procedente de la cartografía digital de Lima y Callao. Esta información fue convertida en formato .shp para poder ser representada gráficamente mediante el uso del Sistema de Información Geográfica ArcGis 9.2.

\section{Distribución espacial de las variables urbanas:}

-Uso del suelo: Se empleó los mapas de zonificación urbana de la ciudad Lima - Callao y se convirtió la información digital al formato shp para poder ser representada gráficamente mediante el uso del Sistema de Información Geográfica ArcGis desktop 9.2. La categorización del uso de suelo empleada, para caracterizar el área de estudio, fue la siguiente: (a) uso residencial, (b) uso comercial, (c) uso industrial, (d) uso de equipamiento, (e) áreas verdes y (f) suelo sin uso.

- Densidad poblacional: Se digitalizó la información de densidad poblacional obtenida a nivel distrital mediante el uso del software ArcGis 9.2, lo cual permitió la representación gráfica espacial.

- Densidad urbana o edificada: La categorización de la densidad urbana se basó en los siguientes criterios, mencionados por Pagliarini (2008): (a) alta densidad de edificación, (b) media densidad de edificación y (c) baja densidad de edificación. Esta información fue categorizada utilizando los mapas catastrales de Lima Callao y usando el Software Google Earth, apoyándose de las anotaciones realizadas en campo durante el recorrido de los transectos. Posteriormente, mediante el uso del ArcGis desktop 9.2, se realizó la representación gráfica de esta variable condicionante.

\section{Análisis de la influencia de las variables condicionantes en el desarrollo de la isla de calor urbana}

Se consideró como variables condicionantes a cada una de las categorías del uso del suelo y de densidad urbana.

Se cuantificó el porcentaje del área ocupada por cada una de estas variables (Tabla 2), dentro de un área de influencia circular con un radio de $300 \mathrm{~m}$ alrededor de cada punto de muestreo, siendo un total 189 puntos.

Posteriormente, se empleó los gráficos de dispersión (relación entre dos variables) para conocer la relación o asociación de cada una de estas variables con la isla de calor estandarizada y dado que de este tipo de gráfico no se

Tabla 2. Categorías del uso del suelo y de la densidad urbana

\begin{tabular}{|l|l|}
\hline \multicolumn{2}{|c|}{ Variables condicionantes } \\
\hline \multirow{4}{*}{ Uso del suelo } & Uso residencial \\
\cline { 2 - 2 } & Uso comercial \\
\cline { 2 - 2 } Uso industrial \\
\cline { 2 - 2 } Densidad urbana & Uso de equipamiento \\
\cline { 2 - 2 } & Áreas verdes \\
\cline { 2 - 2 } & Suelo sin uso \\
\hline & Densidad urbana alta \\
\cline { 2 - 2 } & Densidad urbana media \\
\cline { 2 - 2 } & Densidad urbana baja \\
\hline
\end{tabular}


puede inferir el grado de relación, se halló la correlación existente entre ellas. De acuerdo con los valores de correlación, se consideraron las variables más significativas para incorporarlas junto con las variables de densidad poblacional, distancia a fuentes de agua y altitud a un análisis de regresión múltiple por pasos. Este análisis de regresión múltiple consiste en determinar la relación y grado de asociación entre una variable dependiente (temperatura) y sus supuestas variables explicativas, estableciéndose pasos en los que se introduce cada una de estas variables para determinar el grado de correlación que poseen con la variable dependiente.

Finalmente, para determinar la influencia que tienen las variables condicionantes en la isla de calor urbana, para cada hora analizada, se empleó el análisis de regresión múltiple por pasos, el cual, como se mencionó, incorpora al modelo matemático las variables de acuerdo a la correlación que presentan con la distribución espacial del fenómeno analizado. Este análisis se realizó con la finalidad de encontrar aquellas variables que determinan mejor la presencia del fenómeno estudiado. Las variables introducidas en el modelo se encontraron en su forma estandarizada, para que así todas las variables se encuentren en la misma escala de medida.

\section{Resultados y discusión}

\section{Identificación de la presencia de la isla de calor}

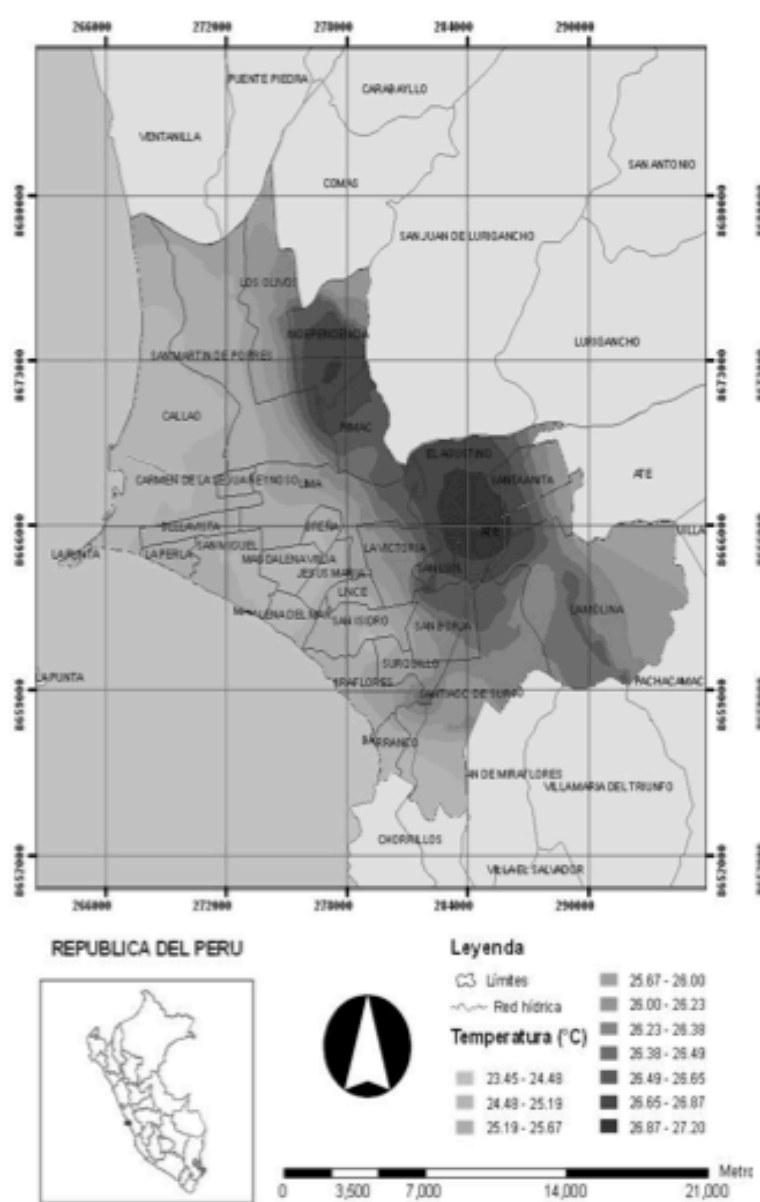

Los mapas térmicos promedio muestran la existencia de la isla de calor en el área de estudio, la cual se detectó en las dos horas analizadas. La isla de calor presentó una clara forma concéntrica, con dos núcleos a las 16:00 h y con un núcleo a las 19:00 h. Específicamente, se observó que el mapa térmico de la temperatura promedio para las 16:00 h tuvo una distribución paralela a la línea de la costa, como lo indica Quezada (1987), y mostró la presencia de dos núcleos cálidos concéntricos con temperaturas que iban desde los $26,7{ }^{\circ} \mathrm{C}$ hasta los $27,2{ }^{\circ} \mathrm{C}$. El primer núcleo térmico abarcó, predominantemente, los distritos de Independencia y Rímac, mientras que el segundo núcleo se encontró predominantemente en los distritos de Ate Vitarte, Santa Anita, El Agustino y San Luis. Mientras que para las 19:00 h, la distribución de la temperatura del aire presentó una configuración distinta, se observó la presencia de un núcleo cálido en los distritos del Rímac, Independencia y en parte de los distritos de Los Olivos y San Martín de Porres.

Determinación de la intensidad de la isla de calor urbana.

De acuerdo con los resultados obtenidos (Tabla 3), la máxima intensidad de la isla de calor urbana a las 16:00h fue ligeramente mayor que a las 19:00 h; por lo que en ambos días de monitoreo la intensidad de la isla de calor fue predominantemente moderada.

Determinación de la distribución espacial de las variables condicionantes de la isla de calor urbana.

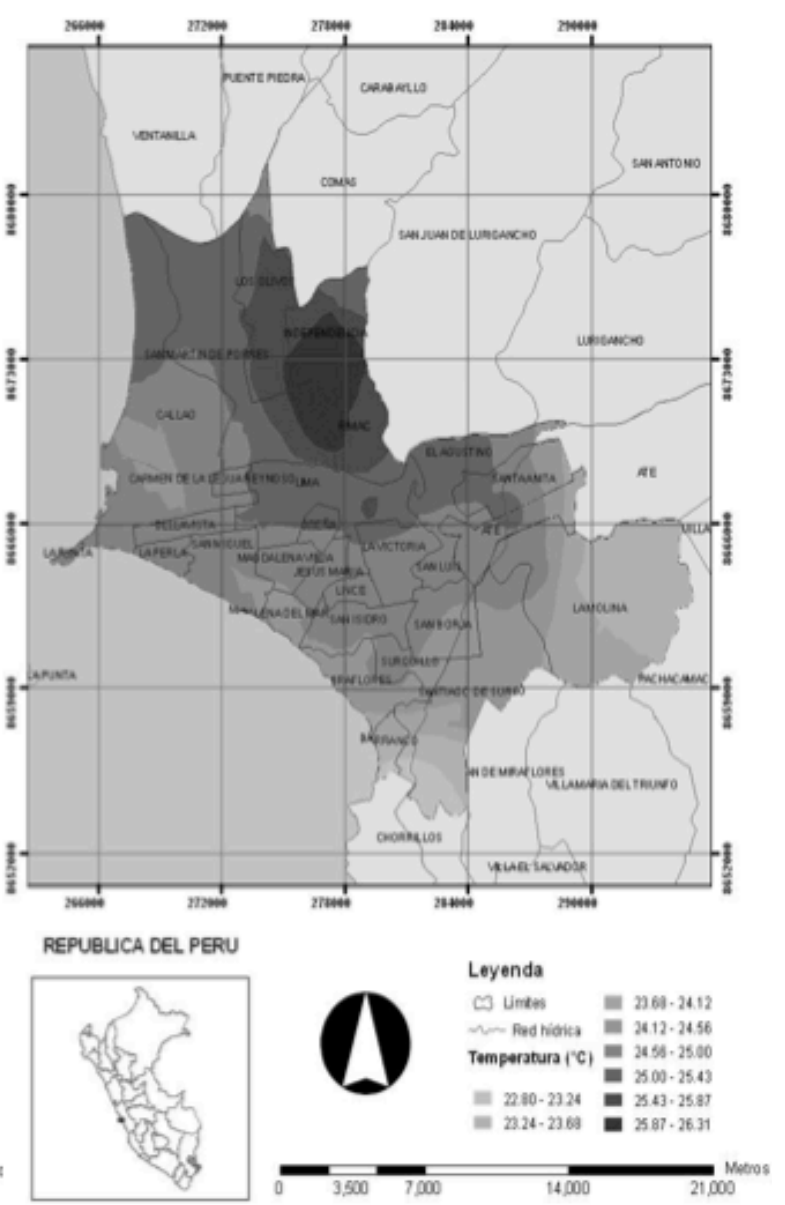

Figura 3. Mapa térmico del área de estudio a las 16:00 h (izq.) y a las 19:00 h (der.) 
Tabla 3. Valores de la intensidad promedio de la isla de calor urbana

\begin{tabular}{|c|c|c|c|c|}
\hline Hora & \multicolumn{2}{|c|}{ Temperatura } & $\begin{array}{c}\text { Intensidad } \\
\text { de la ICU }\end{array}$ & Categoría \\
\hline $16: 00$ & 27.2 & 23.5 & 3.7 & Moderada \\
\hline $19: 00$ & 26.3 & 22.8 & 3.5 & Moderada \\
\hline
\end{tabular}

La densidad poblacional promedio, número de habitantes por cada kilometro cuadrado en el área de estudio, es de $14.073 \mathrm{hab} / \mathrm{km}^{2}$. El mapa de densidad poblacional indica que la densidad mínima es de $2.015 \mathrm{hab} / \mathrm{km}^{2}$ en la Molina y la máxima es de $25.804 \mathrm{hab} / \mathrm{km}^{2}$ en Surquillo. Los distritos con menores valores de densidad poblacional, menores a $12.045 \mathrm{hab} / \mathrm{km}^{2}$, se encuentran ubicados predominantemente al borde del litoral costero y al sur-este del área de estudio, mientras que los que poseen mayores valores, superiores a $12.044 \mathrm{hab} / \mathrm{km}^{2}$, se concentran al nor-este y en el centro del área.

El área de estudio que abarca 27 distritos de la ciudad Lima se encuentra ubicado, en gran parte, a una altitud por debajo de los 250 metros, encontrándose dentro de este rango ( $0 \mathrm{msnm}-250 \mathrm{msnm})$ todos los distritos contiguos a la línea costera hasta alcanzar las zonas de los siguientes distritos: Independencia, Rímac, El Agustino, Ate Vitarte, La Molina y Santiago de Surco. A partir de los distritos mencionados, la variación de la altitud se incrementa conforme se va alejando del Océano Pacífico hasta unos 600 metros aproximadamente, exceptuando al distrito de La Molina.

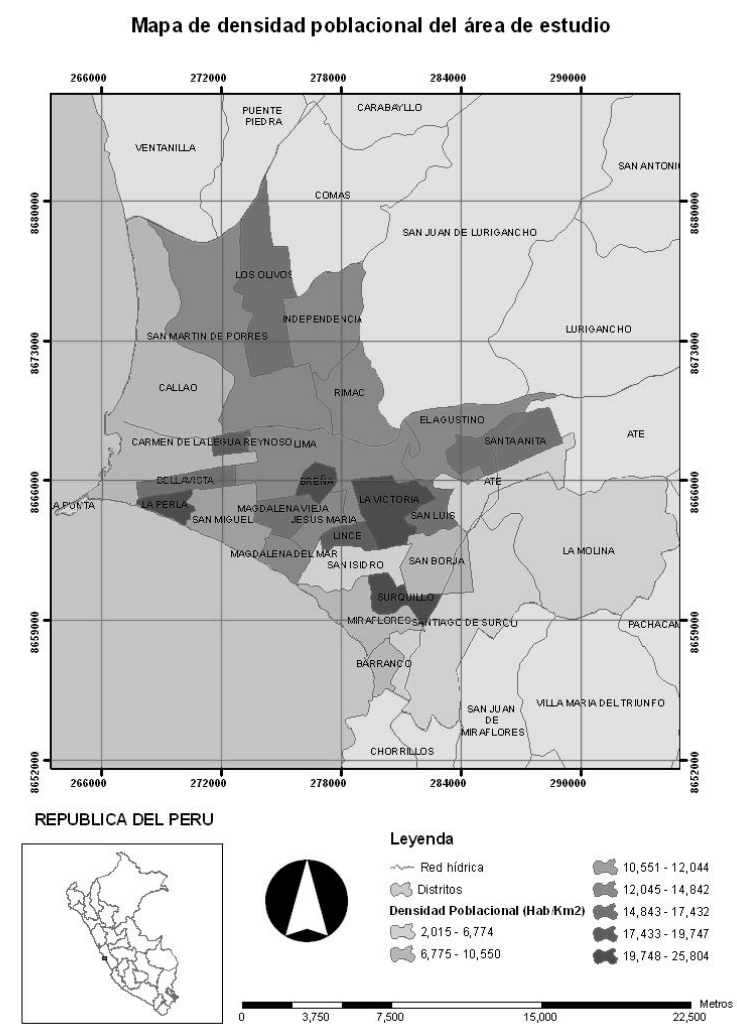

Figura 4. Densidad poblacional del área de estudio
El área de estudio se encuentra en las cuencas de los ríos Rímac y Chillón, por lo que los principales cuerpos de agua que influyen en esta zona son: el Océano Pacífico,

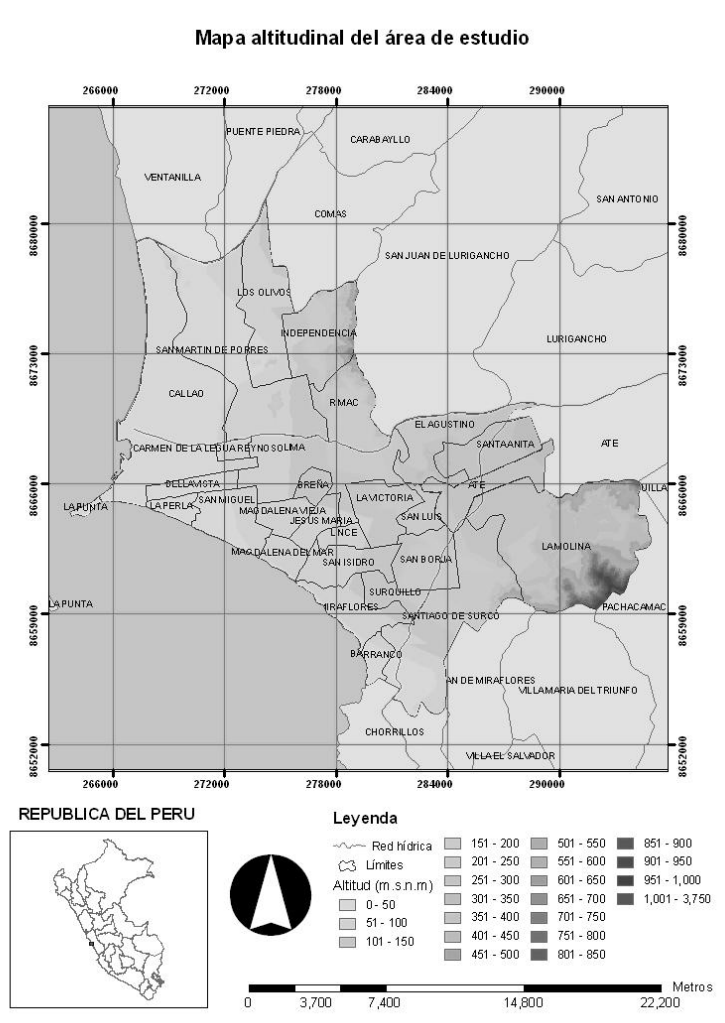

Figura 5. Altitud del área de estudio

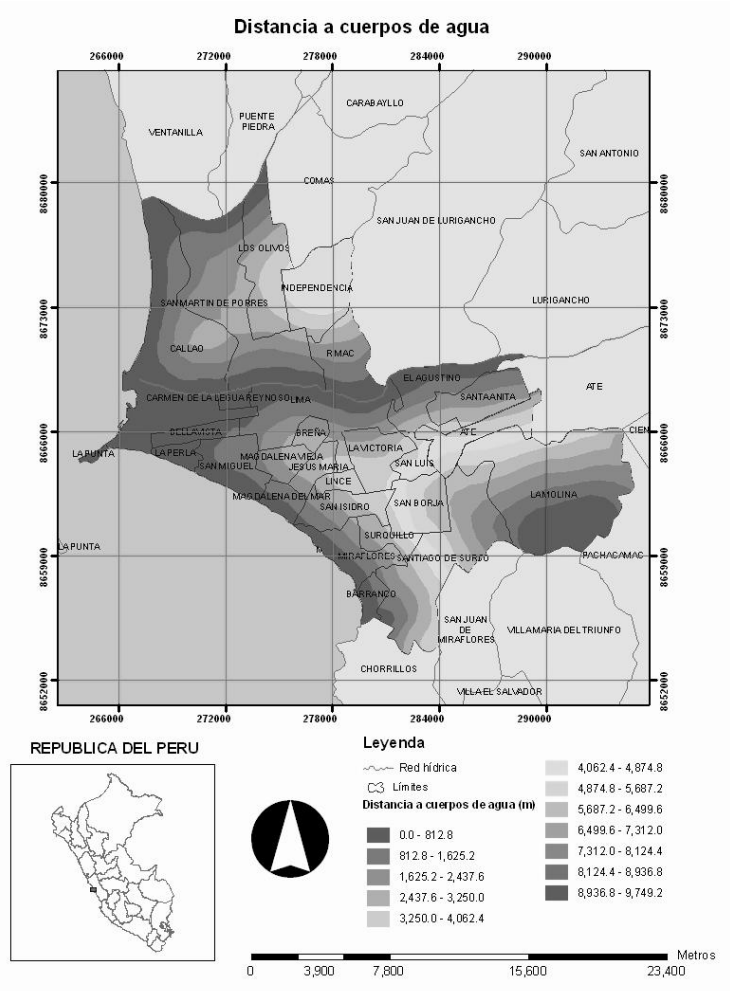

Figura 6. Distancia a fuentes de agua en el área de estudio 
y los ríos mencionados. El río Rímac recorre los distritos de El Agustino, Lima, Rímac, San Martín de Porres, Carmen de la Legua Reynoso y el Callao, mientras que el río Chillón limita con los distritos de Los Olivos, San Martín de Porres y Callao. En la Fig. 6 se observa la distribución de las distancias a los cuerpos de aguas, siendo los distritos de Independencia, Ate Vitarte, La Molina, San Luis, San Borja y parte de Santiago de Surco los más distantes a los ríos y al Océano Pacífico, con distancias que aproximadamente van desde los cuatro a los diez kilómetros.
Se observa que el área de estudio presenta un uso de suelo muy variado (Fig. 7), donde se encuentran mezclados, dentro de la trama urbana, los usos: residencial, comercial, industrial, equipamiento y áreas verdes. Esto se debe a que la ciudad no ha crecido en base a una planificación urbana, muy por el contrario, su desarrollo ha sido en función de las necesidades y crecimiento de la población.

En el mapa de densidad urbana (Fig. 8) se aprecia que esta es principalmente media y alta dentro de la trama urbana, mientras que por otra parte, en la zona periférica

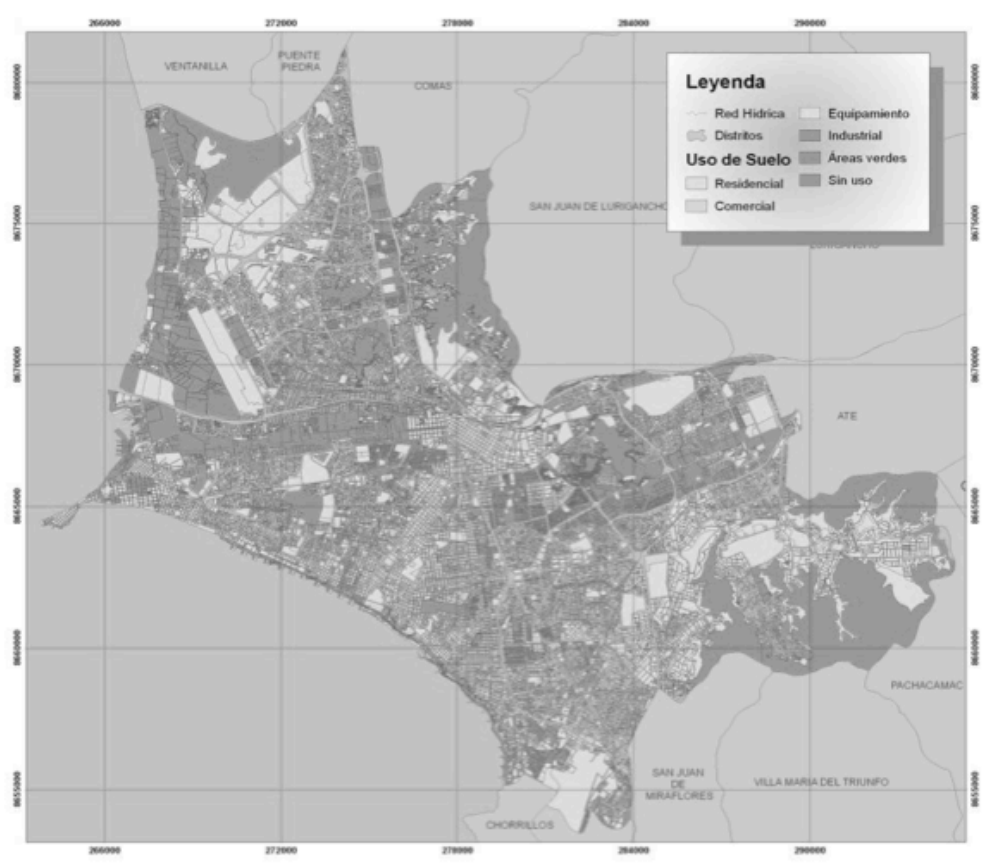

Figura 7. Uso de suelo del área de estudio

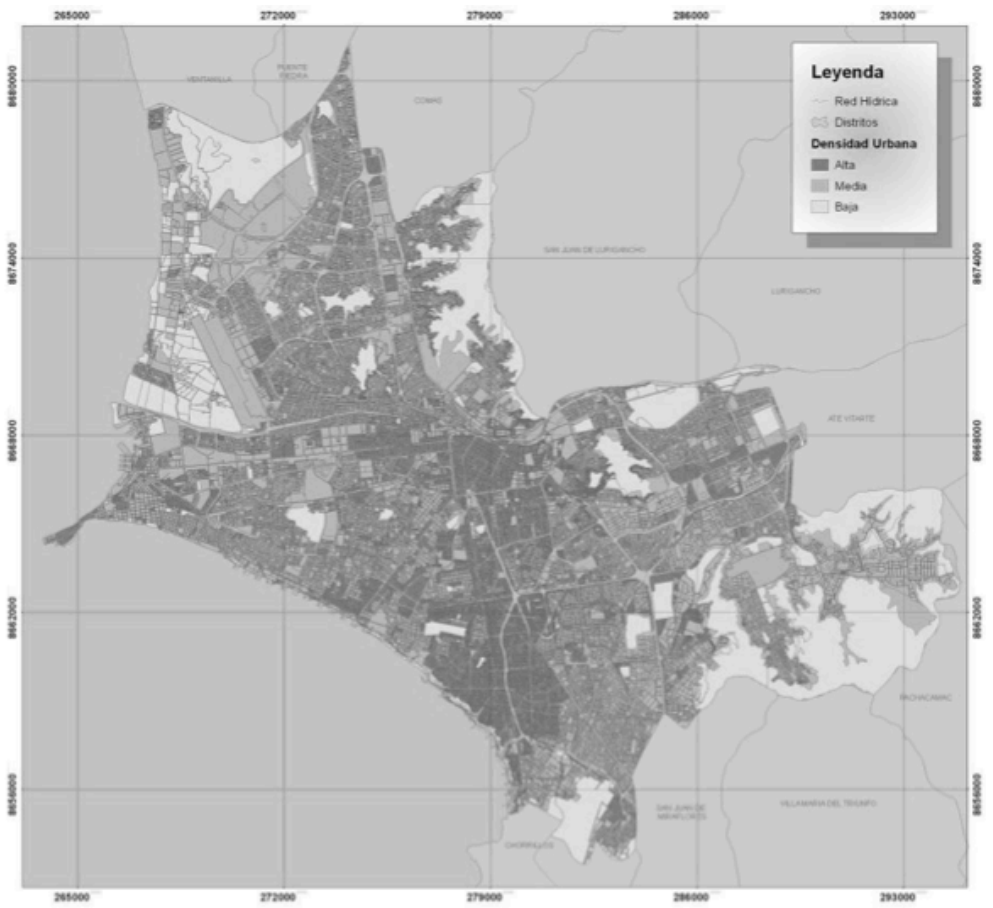

Figura 8. Densidad urbana del área de estudio 
del área de estudio, existe la presencia de zonas con una densidad urbana baja.

\section{Análisis de la influencia de las variables condicionantes en el desarrollo de la isla de calor urbana}

El análisis de regresión múltiple por pasos realizado entre las variables condicionantes y la isla de calor urbana determinó las variables que condicionaron la presencia del fenómeno en cada hora analizada. Los resultados (Tabla 4) confirman que para las $16: 00 \mathrm{~h}$, las variables condicionantes que explicaron el $61,0 \%$ de la varianza espacial de la isla de calor en el área de estudio fueron: (a) la altitud, (b) la densidad urbana baja, (c) el uso residencial del suelo, (d) la densidad urbana media y (e) la densidad poblacional.

La variable más importante fue la altitud, explicando por sí sola el 53,0 \% de la varianza espacial de la isla de calor en el área de estudio. La segunda variable, densidad urbana baja, explicó un 4,0\% de la variabilidad del fenómeno, seguida por el uso residencial del suelo que explicó un 1,0 $\%$; posteriormente, se tiene la variable densidad urbana media que explicó un 2,0\% y, finalmente, la densidad poblacional que explicó también un 1,0 \% de la varianza total.

La Tabla 5, para las 19:00 horas, muestra las variables condicionantes que explicaron el $44,0 \%$ de la varianza espacial de la isla térmica, las cuales fueron: (a) la distancia a fuentes de agua, (b) la densidad urbana media, (c) el uso residencial del suelo, (d) la densidad urbana baja y (e) la altitud. Las variables que no se incluyeron en el modelo de regresión múltiple por pasos fue porque se encontraron muy relacionadas con otras variables las cuales, específicamente, fueron: las áreas verdes, la densidad poblacional, el uso industrial del suelo y el suelo sin uso.

El factor condicionante más importante, para las 19:00 horas, fue la distancia a fuentes de agua, explicando por sí sola el $22,0 \%$ de la varianza espacial de la isla de calor. La

Tabla 4. Resultados del análisis de regresión múltiple entre la isla de calor y los diferentes factores condicionantes para las 16:00 h

\begin{tabular}{|c|c|c|c|c|c|}
\hline Modelo & $\begin{array}{c}\text { Coeficiente de } \\
\text { Correlación Múltiple } \\
\text { (R Múltiple) }\end{array}$ & $\begin{array}{c}\text { Coeficiente de } \\
\text { Determinación } \\
\text { Múltiple } \\
\left(\mathbf{R}^{2} \text { Múltiple) }\right.\end{array}$ & $\mathbf{R}^{\mathbf{2}}$ Ajustado & Error ST. & $\begin{array}{c}\text { Cambio } \\
\text { en } \mathbf{R}^{2}\end{array}$ \\
\hline 1 & 0.73 & 0.53 & 0.53 & 0.69 & 0.53 \\
\hline 2 & 0.75 & 0.57 & 0.56 & 0.66 & 0.04 \\
\hline 3 & 0.76 & 0.58 & 0.57 & 0.65 & 0.01 \\
\hline 4 & 0.77 & 0.60 & 0.59 & 0.64 & 0.02 \\
\hline 5 & 0.78 & 0.61 & 0.6 & 0.63 & 0.01 \\
\hline
\end{tabular}

Modelo 1 Altitud.

Modelo 2 Altitud, Densidad urbana baja.

Modelo 3 Altitud, Densidad urbana baja, Uso residencial del suelo.

Modelo 4 Altitud, Densidad urbana baja, Uso residencial del suelo, Densidad urbana media.

Modelo 5 Altitud, Densidad urbana baja, Uso residencial del suelo, Densidad urbana media, Densidad poblacional.

Tabla 5. Resultados del análisis de regresión múltiple entre la isla de calor y los diferentes factores condicionantes para las 19:00 horas

\begin{tabular}{|c|c|c|c|c|c|}
\hline Modelo & $\begin{array}{c}\text { Coeficiente de } \\
\text { Correlación Múltiple } \\
\text { (R Múltiple) }\end{array}$ & $\begin{array}{c}\text { Coeficiente de } \\
\text { Determinación } \\
\text { Múltiple } \\
\mathbf{( R}^{2} \text { Múltiple) }\end{array}$ & $\mathbf{R}^{2}$ Ajustado & Error ST. & $\begin{array}{c}\text { Cambio } \\
\text { en }^{2}\end{array}$ \\
\hline 1 & 0.47 & 0.22 & 0.22 & 0.88 & 0.22 \\
\hline 2 & 0.53 & 0.28 & 0.27 & 0.85 & 0.06 \\
\hline 3 & 0.59 & 0.35 & 0.34 & 0.81 & 0.07 \\
\hline 4 & 0.63 & 0.40 & 0.39 & 0.78 & 0.05 \\
\hline 5 & 0.65 & 0.43 & 0.41 & 0.79 & 0.03 \\
\hline
\end{tabular}

Modelo 1 Distancia a fuentes de agua.

Modelo 2 Distancia a fuentes de agua, Densidad urbana media.

Modelo 3 Distancia a fuentes de agua, Densidad urbana media, Uso residencial del suelo.

Modelo 4 Distancia a fuentes de agua, Densidad urbana media, Uso residencial del suelo, Densidad urbana baja.

Modelo 5 Distancia a fuentes de agua, Densidad urbana media, Uso residencial del suelo, Densidad urbana baja, Altitud. 
segunda variable, que explicó el 6,0\% de la variabilidad de la isla térmica, fue la densidad urbana media, seguida por el uso residencial del suelo, que explicó un 7,0,\% de la varianza total, luego seguida por la densidad urbana baja, influenciando en un $5,0, \%$, y finalmente por la altitud que explicó el 3,0 \% de la varianza espacial.

Las ecuaciones de la Tabla 6 muestran los signos de correlación de los factores condicionantes con respecto a la isla de calor. Este fenómeno tuvo una relación directa o positiva con la altitud, la densidad urbana media y la densidad poblacional a las 16:00 h, mientras que a las 19:00 $\mathrm{h}$ se relacionó positivamente con la altitud y la densidad urbana media. Esto quiere decir que el aumento de dichas variables significa un incremento en la intensidad de la isla de calor y viceversa. Las correlaciones son negativas para la densidad urbana baja y el uso residencial del suelo a las 16:00 h y a las 19:00 h; son negativas la distancia a fuentes de agua, la densidad poblacional, la densidad urbana baja y el uso residencial del suelo, es decir, la disminución de dichas variables provoca un aumento en la intensidad de la isla de calor urbana.

Los resultados de la Tabla 6 evidencian que la altitud es una variable que condiciona positivamente la presencia de la isla de calor urbana a las 16:00 y 19:00 h. Esta variable condicionante fue la más importante a las 16:00 horas, ya que fue responsable en más de un 50,0\% de la variabilidad espacial de la isla de calor urbana en el área de estudio, mientras que su influencia en la ICU en horas nocturnas no fue tan relevante. Se sabe que la temperatura disminuye conforme la altitud se incrementa, sin embargo esta relación no se observó en los resultados estadísticos obtenidos para las dos horas analizadas, lo cual podría explicarse por la presencia de la inversión térmica que caracteriza a la ciudad de Lima y que origina el aumento de la temperatura conforme la altitud se incrementa. Sin embargo, se resalta que con los datos trabajados en el presente estudio no se puede afirmar la presencia de la inversión térmica para los días 12 y 19 de marzo del 2009.

La densidad urbana baja, analizada en 189 puntos de muestreo, es un factor que condiciona negativamente a la isla de calor para las dos horas analizadas, sin embargo fue una variable que presentó poca relevancia al explicar la presencia del fenómeno en el área de estudio. Para la investigación se consideró como áreas de densidad urbana baja las que no tienen un uso de suelo y las que son ocupadas por áreas verdes, por lo que se les atribuye, a estas últimas, el efecto negativo en la presencia de la isla de calor actuando como focos de frescor.

Otro factor condicionante de la isla de calor urbana, para las 16:00 y 19:00 h, es el uso residencial del suelo. Esta variable urbana es un factor que influyó negativamente en la presencia e intensidad de la isla de calor presentando una pequeña influencia en el fenómeno, la cual se explica por el hecho de que las zonas residenciales se encuentran colindando, en su mayoría, con pequeños parques urbanos.

Por otra parte, la densidad urbana media, variable urbana, influye positivamente en la génesis e intensidad de la isla de calor, a las 16:00 y 19:00 horas. El conjunto de edificaciones con esta característica almacena el calor recibido por la radiación solar durante el día, para luego liberar la radiación en onda larga hacia la atmósfera inmediata, calentando así lentamente la temperatura del aire originando que la variación térmica entre estas zonas y las áreas con menos presencia de edificaciones se vea incrementada.

La densidad poblacional favorece la aparición del fenómeno en el área de estudio a las 16:00 h, explicando el 1,0\% de la variación espacial de la isla de calor urbana. Este resultado evidenció la relación positiva mencionada por Oke (1973), citado por Moreno (1999), el cual propuso que la isla térmica se ve intensificada cuando

Tabla 6. Resultados de la constante y coeficientes estandarizados de la ecuación de regresión múltiple para las dos horas analizadas

\begin{tabular}{|c|l|c|}
\hline Horas & \multicolumn{1}{|c|}{ Variables del modelo } & Coeficientes estandarizados \\
\hline \multirow{5}{*}{$16: 00$} & Constante & 0.000 \\
\cline { 2 - 3 } & Altitud & 0.698 \\
\cline { 2 - 3 } & Densidad urbana baja & -0.198 \\
\cline { 2 - 3 } & Uso residencial del suelo & -0.200 \\
\cline { 2 - 3 } & Densidad urbana media & 0.202 \\
\cline { 2 - 3 } & Densidad poblacional & 0.118 \\
\hline \multirow{5}{*}{$19: 00$} & Constante & 0.000 \\
\cline { 2 - 3 } & Distancia a fuentes de agua & -0.581 \\
\cline { 2 - 3 } & Densidad urbana media & 0.331 \\
\cline { 2 - 3 } & Uso residencial del suelo & -0.259 \\
\cline { 2 - 3 } & Densidad urbana baja & -0.224 \\
\cline { 2 - 3 } & Altitud & 0.186 \\
\hline
\end{tabular}


existe un mayor número de población.

La distancia a fuentes de agua condiciona el fenómeno negativamente a las 19:00 h, explicando el 22,0\% de la variabilidad espacial de la isla de calor urbana en el área de estudio. Esta relación es explicada por el efecto termorregulador del océano y ríos, el cual disminuye las variaciones térmicas de las zonas adyacentes, por lo que el enfriamiento de dichas áreas se realiza con mayor lentitud que las áreas más alejadas. Sin embargo, en la zona sur-este del área de estudio, no sucede lo señalado y más bien se observa que el núcleo sur de la ICU, detectada a las 16:00 h, desaparece. Este hecho se debe a que los distritos de La Molina, Santiago de Surco y San Borja tienen presencia de muchas áreas con densidad urbana baja y con un uso residencial del suelo, caracterizadas por la baja densidad de edificación y presencia de áreas verdes, lo cual afecta negativamente al desarrollo de la ICU.

Finalmente, el uso industrial del suelo es un factor que favorece la presencia de la isla térmica a las 19:00 h, teniendo una relación positiva con el fenómeno, es decir, a mayor presencia de áreas con un uso industrial del suelo, mayor intensidad de la isla de calor urbana. Se sabe que en estas zonas se generan concentraciones significativas de gases de efecto invernadero, provenientes de los procesos industriales, las cuales tienden a elevar la temperatura del aire al no permitir la liberación del calor hacia la parte superior de la atmósfera. La importancia de este factor no fue tan significativa a la hora de explicar el fenómeno, influenciando solamente un 4,0\% en la distribución espacial de la isla térmica.

\section{Conclusiones}

En la ciudad de Lima se evidencia la presencia de la isla de calor urbana. A las 16:00 h se identificó la presencia de dos núcleos cálidos, mientras que a las 19:00 h se identificó la presencia de solamente un núcleo cálido ubicado al nor-este del área de estudio. La intensidad de la isla de calor urbana fue moderada para las dos horas de análisis, presentando valores de $3,7^{\circ} \mathrm{C}$ a las $16: 00$ h y 3,5 ${ }^{\circ} \mathrm{C}$ a las 19:00 h.

La distribución espacial de la isla térmica muestra que este fenómeno, a las 16:00 h, presenta dos núcleos ubicados al este del área de estudio, en los distritos más alejados al océano Pacífico; mientras que a las 17:00 h la ICU se localiza en los distritos localizados en el noreste del área de estudio, desapareciendo el núcleo sureste, debido a que los distritos de La Molina, Santiago de Surco y San Borja tienen presencia de muchas áreas con densidad urbana baja y con un uso residencial del suelo, caracterizadas por la baja densidad de edificación y presencia de áreas verdes, afectando negativamente al desarrollo de la ICU.

Los resultados estadísticos evidenciaron que los factores que explican la intensidad de la isla de calor en la ciudad de Lima no tuvieron la misma importancia en el condicionamiento de la misma. Por un lado, la isla de calor urbana detectada a las 16:00 h se encontró condicionada por la altitud (53\%), la densidad poblacional (1\%), la densidad urbana media (2\%), la densidad urbana baja (4 $\%)$ y el uso residencial del suelo (1\%); los tres primeros factores son los que favorecieron positivamente el desarrollo de la isla de calor. Por otro lado, a las 19:00 $\mathrm{h}$, los factores condicionantes del fenómeno fueron la densidad urbana media (6\%), la altitud (3\%), el uso residencial del suelo $(7 \%)$, la densidad urbana baja $(5$ $\%$ ) y la distancia a fuentes de agua (22\%), favoreciendo los dos primeros, positivamente, el desarrollo de la isla térmica.

\section{Literatura citada}

Cuadrat P.; De la Riva, J.; López, F. y Martí, A. 1993. El medio ambiente urbano en Zaragoza, observaciones de la isla de calor. Anales de Geografía de la Universidad Complutense, 13: 119-130.

Instituto Nacional de Estadística e Informática (INEI). 2007. Perú, Censos nacionales 2007, XI de población y VI de vivienda: Sistema de consulta de principales indicadores demográficos, sociales y económicos. Lima, Perú. Disponible en http://censos.inei.gob.pe/ Censos2007/IndDem/.

Quezada, J.M. 1987. Estudio del confort climático en Lima Metropolitana y Callao. (Requerimiento para optar el título de meteorólogo). Universidad Nacional Agraria La Molina. Lima, Perú.

Sarricolea, P.; Aliste, E.; Castro, E. y Escobedo, C. 2008. Análisis de la máxima intensidad de la isla de calor urbano nocturna de la ciudad de Rancagua (Chile) y sus factores explicativos. Revista de Climatología, 8: 71-84. 